\title{
eSports Pro-Players Behavior During the Game Events: Statistical Analysis of Data Obtained Using the Smart Chair
}

\author{
${ }^{1,2}$ Anton Smerdov, ${ }^{1}$ Evgeny Burnaev, ${ }^{1, *}$ Andrey Somov \\ ${ }^{1}$ Skolkovo Institute of Science and Technology, CDISE, Moscow, Russia \\ ${ }^{2}$ Moscow Institute of Physics and Technology, Moscow, Russia \\ *a.somov@skoltech.ru
}

\begin{abstract}
Today's competition between the professional eSports teams is so strong that in-depth analysis of players' performance literally crucial for creating a powerful team. There are two main approaches to such an estimation: obtaining features and metrics directly from the in-game data or collecting detailed information about the player including data on his/her physical training. While the correlation between the player's skill and in-game data has already been covered in many papers, there are very few works related to analysis of eSports athlete's skill through his/her physical behavior. We propose the smart chair platform which is to collect data on the person's behavior on the chair using an integrated accelerometer, a gyroscope and a magnetometer. We extract the important game events to define the players' physical reactions to them. The obtained data are used for training machine learning models in order to distinguish between the low-skilled and high-skilled players. We extract and figure out the key features during the game and discuss the results.
\end{abstract} ing

Keywords-smart chair, eSports, machine learning, smart sens-

\section{INTRODUCTION}

Nowadays eSports is a rapidly growing industry with more than billion players involved worldwide. The competition among top tier teams is strong and involves, apart from the teams themselves, their coaches, managers and associated scientists. As a result, eSports research has significantly progressed for the last few years. But still, to the best of our knowledge there are few works related to the estimation of eSports athletes performance based on their physical behaviour. The player reactions to the game events can be investigated using Electroencephalography (EEG) [1] or brain waves [2]. Also, the game influence can be evaluated through the computer mouse movements [3].

Another approach for evaluating the athlete performance is based on the game statistics. Shim et al. research [4] is devoted to the calculation of Kill/Death/Assist (KDA), Kill Death Ratio (KDR) for a player in a first-person shooter game and predicting them for the next game rounds. Firstperson shooter research is reported in [5] where the authors analyze the dependence between the player skill and the data collected from the keyboard and mouse logs. On top of that there are works presenting the most important factors for players in Multiplayer Online Battle Arena (MOBA) [6],
[7] and Massively Multiplayer Online Role-Playing Game (MMORPG) genres [8], [9].

Research into eSports include (i) affective computing [10], (ii) prediction related research [11], and (iii) social structures in teams [12]. Research in these areas suffers from purely theoretical research without proper experimental work with the professional eSports athletes. Indeed, data collection followed by modelling and interpretation tasks has the potential to forster the eSports research and make it practically feasible.

In this work, we use a smart chair for data collection and further analysis of eSports athletes behaviour. Smart chairs have already been used in the unobtrusive sensing applications: the pressure sensors embedded in a chair provide sufficient information about the sedentary patterns [13], [14]. This information can be used for making classification of the user activity [15], e.g. talking, coughing, eating, as well as stress detection [16]. Another straightforward application is the user posture detection. Authors in [17] report on the posture detection using the tilt sensors in addition to the pressure sensors. A similar method is used in [18] to access the user experience through the smart chair, and, in [19] to build an occupancy detection system.

Another application of the smart chair concept is the measurement of vital signs based on the heart related sensors. Authors in [20] and [21] used EMFi sensors integrated in the chair to measure the Ballistocardiogram (BCG). Ahn et al. [22] proposed the electrocardiography (ECG) method for the measurement of heart rate with the sensors embedded in a chair.

A contribution of our paper is the collection of eSports data using the smart chair and further data analysis using machine learning algorithms. The important part of our work is extracting data about game events and using it to extract more meaningful features.

We managed to build the machine learning models which are able to classify the athletes on the basis of their skills. The best model demonstrates the $77 \%$ accuracy and 0.88 ROC AUC score. The key features have been figured out and ranked.

This paper is organized as follows: in Section II we describe the smart chair platform used for data collection from the professional CS:GO athletes and amateur players. In Section III we present methodology used in this work. Data processing, 


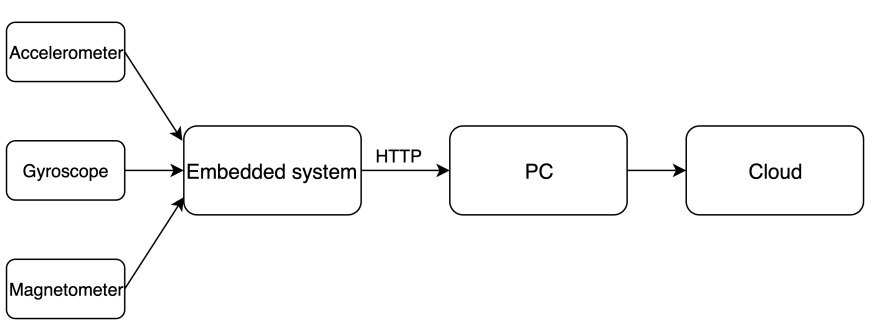

Fig. 1: System architecture.

feature extraction and machine learning algorithms are detailed in Section IV. We provide concluding remarks in Section V.

\section{SMART CHAIR SENSING PLATFORM}

\section{A. System architecture}

The smart chair platform consists of two units: a sensor unit for data collection and a server for data processing. The sensor unit consists of an accelerometer, a magnetometer and a gyroscope. The data are collected by Motion Processing Unit (MPU) 9250 via I2C protocol. This unit is connected to a single-board computer Raspberry Pi 3 . The platform can be further extended by adding some extra sensors, e.g. the pressure and temperature ones. The data are collected every $0.01 \mathrm{~s}$. Upon collecting the data, the sensing unit makes a request and sends them to a server over the WiFi wireless channel. The data are sent to the server via the HTTP protocol every second in the JSON format. The data processing is realized on the server using the machine learning algorithms that are described in more details in the next section. The system architecture is shown in Fig. 1

The experimental testbed is shown in Fig 2, The sensing unit is rigidly fixed at the bottom of the chair not to disturb the game process and capture all of the chair movements. In order to avoid redundant wires from the chair, we power it by a daily rechargeable external battery.

It is important to properly orient sensors properly to simplify further data processing. The axes orientation is shown in Fig. 3 . The axis $z$ is the vertical axis, $y$ is the axis passing through the player and the monitor, $x$ is the axis parallel to the gaming table.

\section{Methodology}

We have invited 9 professional athletes, primarily from the Monolith professional team specializing in CS:GO discipline, and 10 amateur players to take part in the experiment. Before the experiment we informed all the participants about the project and the experiment procedure and collected their written consents. We asked the participants to fill in the questionnaire to make sure that they are in good form and do not take any drugs that might affect the experimental results.

In this work we apply the Retake modification of CS:GO discipline. In the Retake scenario a terrorist team plays against a counter-terrorist team. The terrorist team is made up of 2 players who typically play in a defensive manner: they have a bomb planted on the territory and have to defend it from

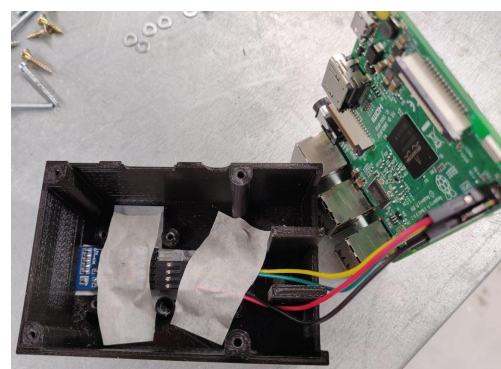

(a) Anatomy of the sensing system.

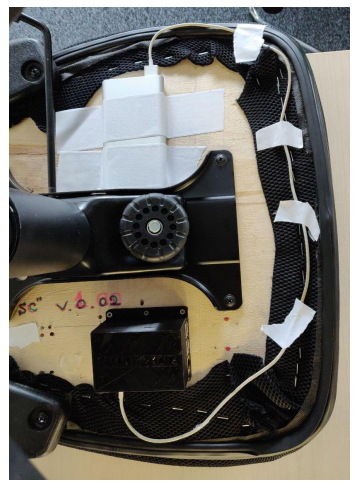

(b) Sensing module fixed on the bottom of a chair.

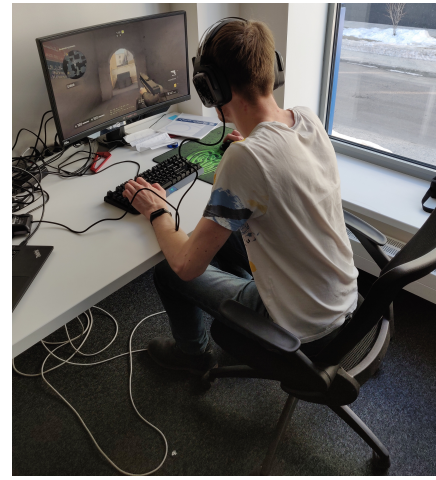

(c) Experimental testbed.
Fig. 2: Sensing system.

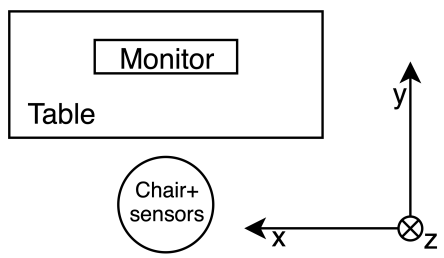

Fig. 3: Axes orientation.

the opposite team. The counter-terrorist team ( 3 players) is to to deactivate the bomb or, alternatively, kill their 'enemies' (the opposite team). The game user interface shows the bomb location on the map in the beginning of each round which lasts for approximately $40 \mathrm{~s}$ (there are 12 rounds altogether). The players have to buy the same set of weapons for each round. The Retake scenario is to be played continuously without any breaks between the rounds.

\section{DATA ANALYSis}

\section{A. Data collection and Pre-processing}

We collected data from 19 persons playing CS:GO. Each gaming session lasts about 35 minutes. As a matter of fact, the player's behavior is sufficiently characterized by a smaller timeseries. That is why, in order to increase the amount of training data, we divide each player's log into 3-minutes sessions, up to 10 non-intersecting sessions per player. As a result, we got 171 labeled timeseries, some of them corresponding to the same players. 


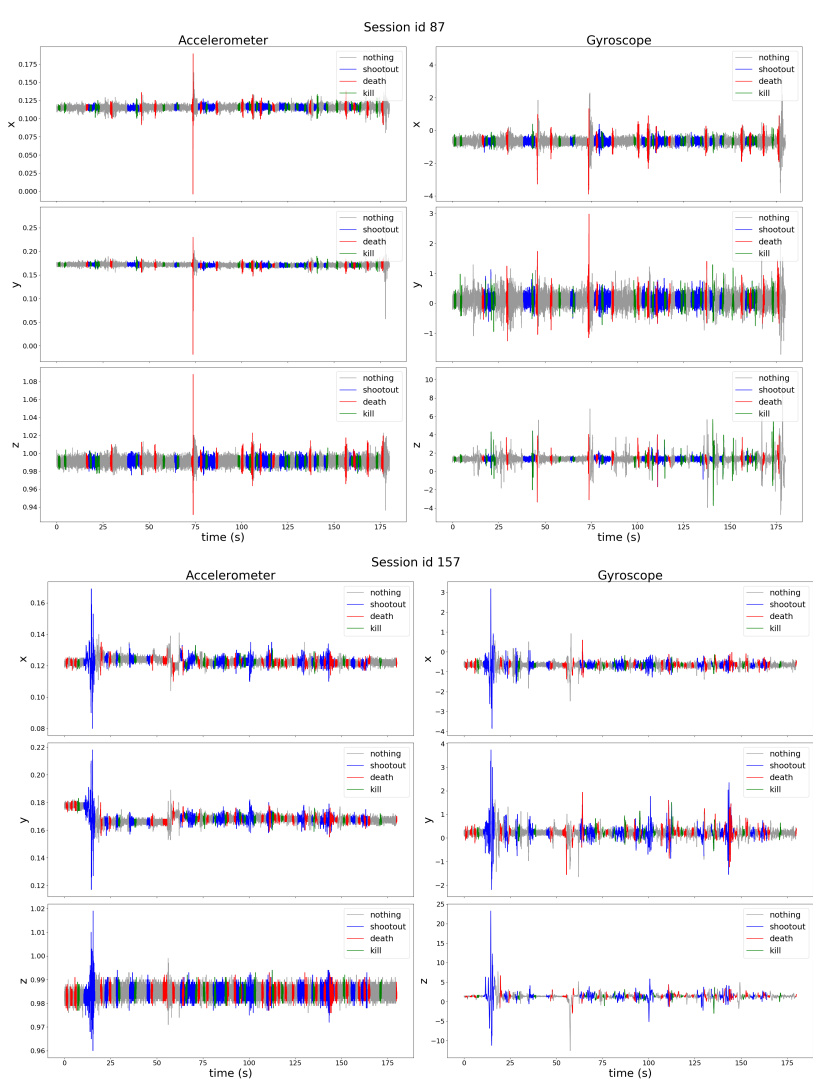

Fig. 4: Examples of the raw data collected from the accelerometer and gyroscope. Colors correspond to game events.

We also extracted the key game events from the gamelogs. For each session, moments of player killing and death were defined. In order to capture the player's behaviour in the tough situations, the 'shootouts' events were extracted. These are the events when the player shoots at least 3 times with the less than 3 seconds delay between the shots.

In our experiment we have three types of data: acceleration from the accelerometer, spatial orientation from the magnetometer and angular velocity from the gyroscope. Examples of the raw data from the accelerometer and gyroscope for a 3minute session are shown in Fig. 4. In order to demonstrate the correlation with the game events we colored the corresponding moments.

It is clear that for most of the time the player does not make lot of movements while sitting on the chair. Some disturbance, however, constantly occurs, sometimes simultaneously with the key game events. Our goal is to check whether the reaction to the game events can describe the player's skill.

While the accelerometer and gyroscope already get acceleration and angular velocity, their raw measurements do not exactly correspond to the movement due to gravity and nonperfect calibration. These measurements are stationary when the player does not move and nonstationary when the player makes movements. It seems more reasonable, therefore, not to use raw data from the sensors, but to apply increment of the time-series. Thus, in order to more effectively extract the disturbance, we calculated the standard deviation within the 1 -second moving window for each of the sensors. The results obtained are shown in Fig. 5. The peaks correspond to the player's active movement on the chair.

\section{B. Feature Extraction}

We formally define the movement on the chair as the moments when the floating standard deviation is 3 times larger than its median value. That means that the player probably changes his posture, twitches or leans on the back of the chair.

Then we obtained data on how often persons actively move on the chair as a proportion of these moments. In order to catch the players' reactions to the game events we calculated how often persons actively move within 1 second after killing or death, or during a shootouts.

Another extracted feature is the portion of time when the person leans on the back of the chair. It is easily calculated using that records from the $z$-component of the accelerometer decreases when the person leans on the back.

In order to assess the player's actual performance during the session, we obtained the Kill Death Ratio (KDR) from the game logs, a popular metric for estimating the player's skill. If the player did not die within the session, we bounded this value by 10 .

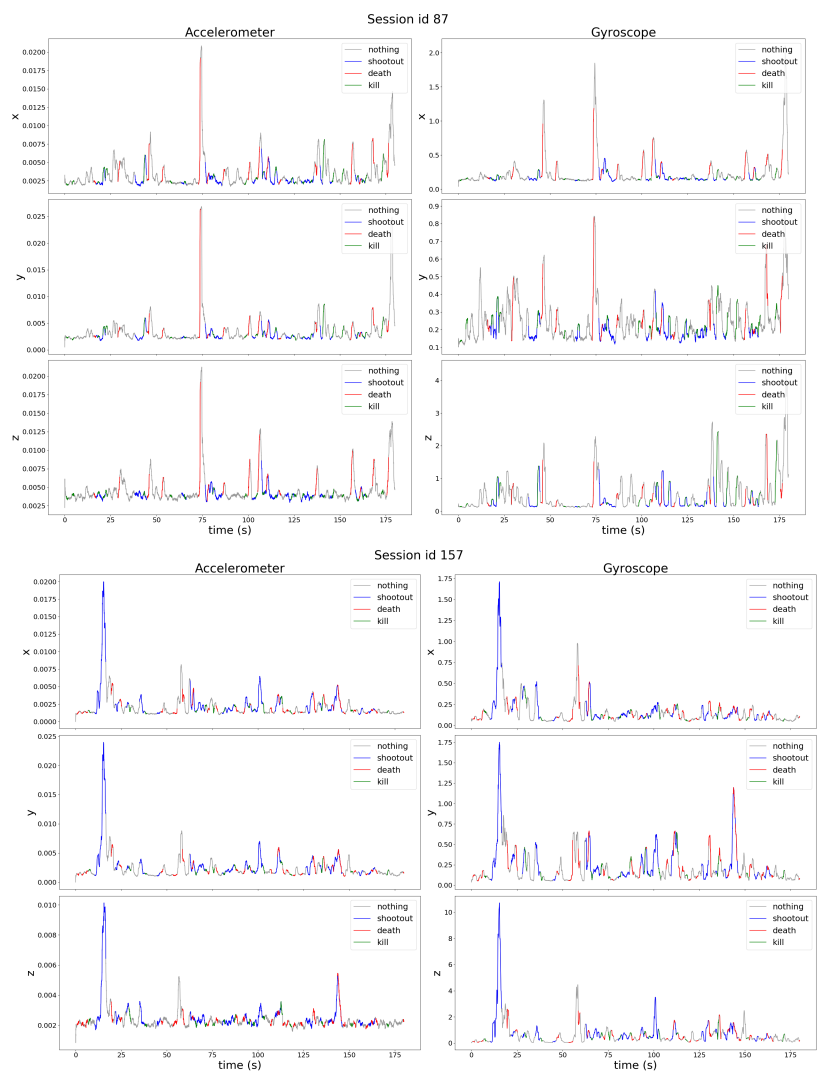

Fig. 5: The floating standard deviation of the sensors data within the 1-second window. Colors correspond to the game events. 
TABLE I: Description of features.

\begin{tabular}{|l|l|}
\hline Feature name & Description \\
\hline$>1000 \mathrm{~h}$ exp & Player has more than 1000 hours of experience. \\
\hline Gender & Gender of a player. 0 is a woman, 1 is a man. \\
\hline Kill Death Ratio & $\begin{array}{l}\text { Number of player's kills divided by number of } \\
\text { player's death in a session }\end{array}$ \\
\hline Age & Age of a player. \\
\hline lean_back & $\begin{array}{l}\text { Portion of time when player leans to the back of } \\
\text { the chair. }\end{array}$ \\
\hline med_acc_x_std & $\begin{array}{l}\text { Median value of the floating standard deviation } \\
\text { within 1-second window for } x \text {-component of ac- } \\
\text { celerometer. The same for } y \text { and } z \text { components } \\
\text { and gyro. }\end{array}$ \\
\hline moving_acc_x & $\begin{array}{l}\text { Proportion of time when floating standard devia- } \\
\text { tion of } x \text { component of accelerometer is 3 times } \\
\text { more than median. In other words, player actively } \\
\text { moves along } x \text { axis. }\end{array}$ \\
\hline moving_gyro_x & $\begin{array}{l}\text { The same as moving_acc_x, but player actively } \\
\text { rotates along } x \text {-axis. }\end{array}$ \\
\hline moving_death_acc_x & $\begin{array}{l}\text { Proportion of time during 1 second after death } \\
\text { when person actively moving. The same for gyro, } \\
\text { other components and events. }\end{array}$ \\
\hline
\end{tabular}

The correlations between the obtained features are shown in Fig. 6 Though we added gender and age of the player for illustrative purposes, we did not use these data in further experiments. The features are described in more detail in Table I]

It is clear from the correlation plot that many features obtained from the chair sensors are highly correlated. It is usually the case for the groups of 6 features and can be explained as follows: moving along one direction oftentimes implies moving along other directions. The heatmap also shows some interesting conclusions from the data the older players, for example, move on a chair less than the younger players, or that men lean to the back of the chair more often than women.

The reason why we used the fact that the player has more than 1000 hours of experience as a target is that the professional high-skilled player definetely has more than 1000 hours of the game time, while the low-skilled player has probably less than 1000 hours of experience. On top of that this target is more informative than that of 100 hours according to the heatmap. As for KDR which is also a good target, it significantly fluctuates within various experiments: people on the server are constantly changing and it would be difficult to build a stable machine learning model for this target. Moreover, KDR, being a continuous metric, has to be somehow splitted into several classes to apply classification. However, a particular variant of such splitting is not easy to justify.

\section{Feature Selection}

In order to figure out the most important factors and to build a stable machine learning model, the feature selection algorithms should be applied. Assuming that each feature monotonically affects the target and considering high correlations between them, the feature selection algorithms based on the linear models can be successfully applied. In particular,
LASSO method can select the most important factors in a linear model (here we temporary switch over to the regression problem instead of the classification). However, a number of the features selected strongly depend on a regularization constant, which is selected manually [23].

LASSO is the method which minimizes the functional:

$$
\frac{1}{2 n}\|y-X w\|_{2}^{2}+\alpha\|w\|_{1},
$$

where $X$ is the design matrix, $y$ is the vector with target values, $w$ is the vector of coefficients, $\alpha$ is the regularization constant.

In order to select the optimal regularization constant $\alpha$ and the most important features, the information criteria, e.g. the Akaike information criterion (AIC) or Bayes Information criterion (BIC), are widely used [24]. These criteria can efficiently select the accurate model which uses some of the most important features. It is achieved by simultaneously penalizing the model for a number of used features and maximizing the likelihood function of the model.

The AIC value for the model is determined by the number of the $k$ parameters (the number of features in our case) and the maximum value of the likelihood function $\hat{L}$ with respect to those parameters:

$$
\mathrm{AIC}=2 k-2 \ln (\hat{L}) .
$$

The BIC is similar, but it penalizes the number of features more significantly:

$$
\mathrm{BIC}=\ln (n) k-2 \ln (\hat{L}),
$$

where $n$ is the number of observations. The smaller both AIC and BIC values are, the better.

The dependence of AIC and BIC w.r.t. $\alpha$ is shown in Fig. 7 The best model according to AIC has 8 features, while according to BIC it has 4 features - and all of them are included in the top- 8 features. These key factors are presented in Table II The negative coefficients correspond to the factors that are intrinsic to the low-skilled players, while the positive coefficients correlate with the features that are specific to the high-skilled players. Coefficients with the higher absolute values are more important.

Interestingly enough, there are no features associated with the event when the player makes a frag. In this situation, apparently all the players react in the same way. Besides, there are no features related to the $z$-component of the accelerometer, perhaps for the reason that the height of the chair is fixed at the beginning of the experiment and does not change further.

\section{Machine Leaning}

In order to estimate the possibility of predicting the player's skill by his/her behaviour on a chair, we built several machine learning models. To obtain correct results models were trained on all sessions of a random half of players and validated on all sessions of other players. for training we used only 8 features selected above. As the target variable $y$ we use the fact that a person has more than 1000 hours experience in CS:GO. 

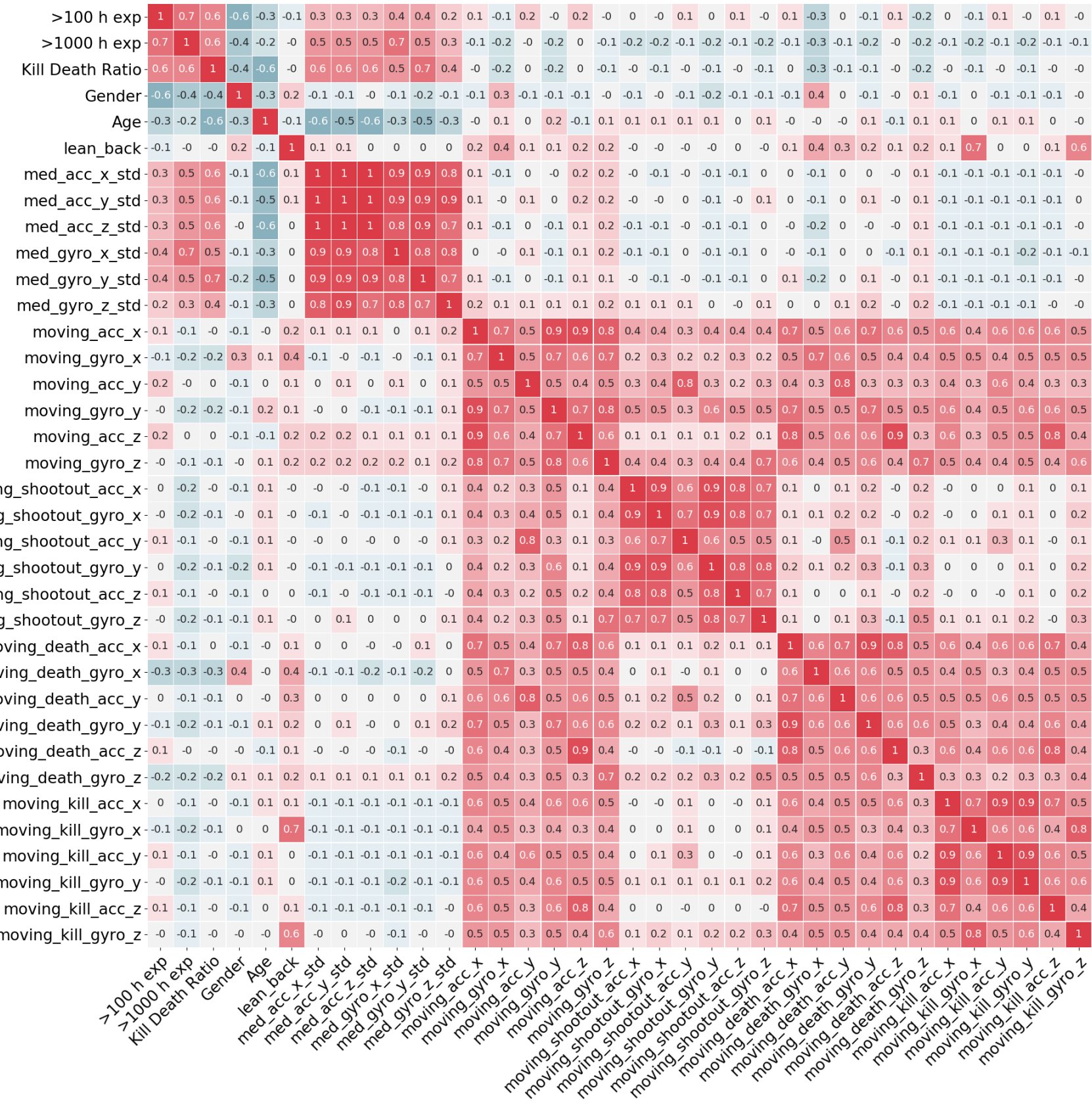

Fig. 6: Correlations between thefeatures.

We applied 6 fundamentally different standard machine learning algorithms with hyperparameters adjusted to our problem:

1) Logistic regression. Simple linear model for classification [25],

2) Support vector machine (SVM) with radial basis functions (RBF) kernel. Nonlinear method for data separation [26],

3) Random forest with 100 estimators and maximum tree depth 2. Plenty of diverse decision trees voting for the optimal class [27],
4) k-nearest neighbors classifier with $\mathrm{k}=3$. Simple algorithm trying to find similar objects in the train data [28],

5) Naive Bayes. This method assumes that the features are independent and estimates their distribution for each target class. Since the data are mostly continuous, we used Gaussian distribution (Gaussian Naive Bayes) [29],

6) Gaussian process. This algorithm implies the probabilistic nature of data and tries to predict the target class using a latent function [30].

To describe methods performance comprehensively we used several evaluation metrics. 


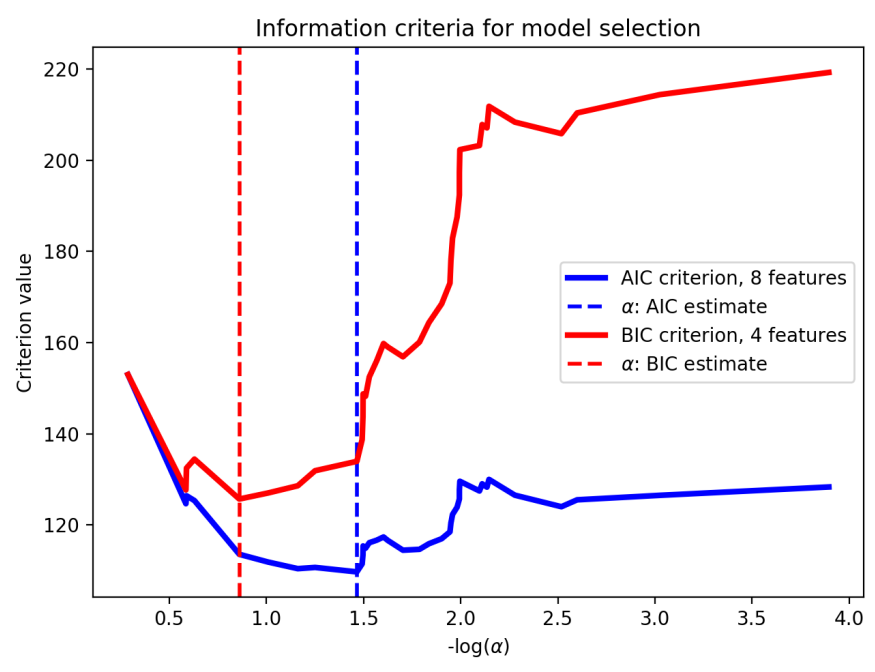

Fig. 7: Dependence of AIC and BIC for LASSO w.r.t. $\alpha$.

TABLE II: Features selected using AIC.

\begin{tabular}{|l|l|l|}
\hline Feature name & Coef. & Possible sense \\
\hline moving_death_gyro_x & -0.17 & $\begin{array}{l}\text { How often after the death player } \\
\text { quickly leans back (or opposite, get } \\
\text { close to a monitor). }\end{array}$ \\
\hline moving_shootout_gyro_z & -0.16 & $\begin{array}{l}\text { How often during the shootout } \\
\text { player spins on the chair. }\end{array}$ \\
\hline moving_death_gyro_y & -0.07 & $\begin{array}{l}\text { After the death player changes a } \\
\text { posture. }\end{array}$ \\
\hline moving_shootout_acc_y & -0.07 & $\begin{array}{l}\text { How often during the shootout } \\
\text { player approaches or move away } \\
\text { from monitor. }\end{array}$ \\
\hline med_gyro_y_std & 0.03 & $\begin{array}{l}\text { How intensely player moves in a } \\
\text { chair. }\end{array}$ \\
\hline moving_acc_x & 0.05 & $\begin{array}{l}\text { How often player moves along the } \\
\text { table. }\end{array}$ \\
\hline moving_acc_y & 0.11 & $\begin{array}{l}\text { How often player approaches or } \\
\text { move away from a monitor. }\end{array}$ \\
\hline med_gyro_x_std & 0.42 & $\begin{array}{l}\text { How intensely player wiggles to a } \\
\text { monitor. }\end{array}$ \\
\hline
\end{tabular}

1) Accuracy, or proportion of right predictions. Simple metric estimating chance of the right prediction. The higher values are, the better.

2) The area under the receiver operating characteristic curve (ROC AUC). It ranges from 0 to 1 with the 0.5 value for random guessing. The higher values are, the better [31].

3) Log Loss, or cross-entropy for binary case. This describes the imperfection of the classification from the information theory view. The lower values are, the better.

Since the number of positive and negative samples is approximately the same, the application of all these metrics is reasonable. For the more precise estimation, each metric was calculated for 1000 different random train/test splits and averaged. The evaluation results for all the algorithms are shown in Table III.

The random forest turned out to be the best performing algorithm, probably because it can catch non-trivial interac-
TABLE III: Scores for the machine learning algorithms.

\begin{tabular}{|c|c|c|c|}
\hline & Accuracy & ROC AUC & Log Loss \\
\hline Logistic regression & 0.71 & 0.86 & 0.60 \\
\hline SVM & $\mathbf{0 . 7 8}$ & 0.85 & 0.84 \\
\hline Random forest & 0.77 & $\mathbf{0 . 8 8}$ & $\mathbf{0 . 4 6}$ \\
\hline k-nearest neighbors & 0.77 & 0.73 & 5.59 \\
\hline Naive Bayes & 0.76 & 0.69 & 5.50 \\
\hline Gaussian process & 0.71 & 0.86 & 0.60 \\
\hline
\end{tabular}

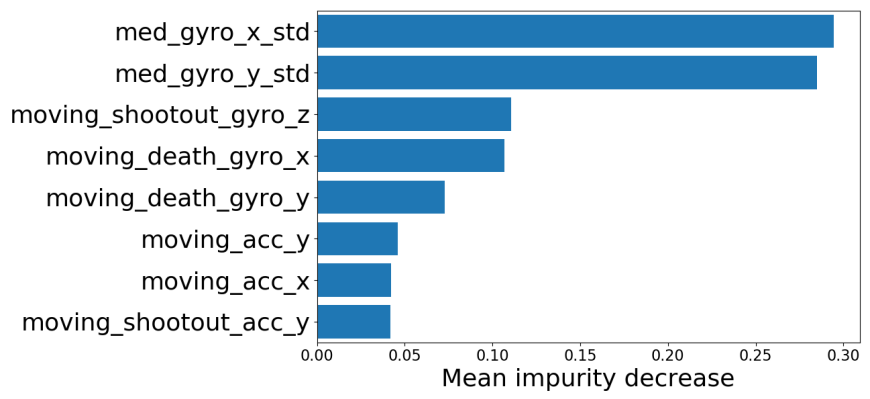

Fig. 8: Feature importance as a mean impurity decrease for random forest.

tions between the features. SVM has a slightly better accuracy, but much worse Log Loss. 0.88 ROC AUC score is an improvement as compared to 0.86 achieved in [32] where information about the game event was not used. It means that we can differentiate the high-skilled player from the low-skilled player with the $78 \%$ accuracy. More important, however, is that this score can be used for the additional estimation of the player skill in professional CS:GO teams in a short period of time.

In order to determine which of the 8 selected features are more essential, we calculated the feature importance for the random forest based on the mean decrease in impurity. These results are more reliable than estimating the feature importance estimation by combination of AIC and LASSO (see Table IIT), for the random forest model can catch nonlinear dependencies and performs better according to Table III Results are shown in Fig. 8

The general activity on the chair turned out to be more important than reactions to game events. Each component from the accelerometer and the gyroscope, except for the $z$ component of the accelerometer, was useful for determining a player's skill.

\section{CONCLUSIONS}

In this work we have proposed the smart chair sensing platform for the collection and analysis of data in eSports. We have processed the raw data from the sensors integrated in the chair and combined them with the key game events. These data have been further engineered into the features that helped us figure out the most important qualities intrinsic to the professional CS:GO athletes. Several machine learning models have been built to assess informativeness of the data. As a result, we have got the accurate algorithm capable 
of predicting the player's skill exclusively on the basis of information received within approximately 3 minutes of his game session.

Future work provides for the more accurate data preprocessing and timeseries segmentation using hidden markov models [33] and anomaly detection approaches [34]. The online prediction of the player's performance shall be carried out using the specific metrics for classification of time-series segments [35] and manifold learning for nonlinear feature extraction [36].

\section{ACKNOWLEDGMENT}

The reported study was funded by RFBR according to the research project No. 18-29-22077 \18.

Authors would like to thank Skoltech Cyberacademy, CS:GO Monolith team and their coach Rustam "TsaGa" Tsagolov for fruitful discussions while preparing this article. Also, the authors thank Alexey "ub1que" Polivanov for supporting the experiments by providing a slot at the CS:GO Online Retake server (http://ub1que.ru).

\section{REFERENCES}

[1] Z. Minchev, G. Dukov, and S. Georgiev, "Eeg spectral analysis in serious gaming: An ad hoc experimental application," Bio Automation, vol. 13, no. 4 , pp. 79-88, 2009.

[2] B. Meneses-Claudio and A. Roman-Gonzalez, "Study of the brain waves for the differentiation of gamers category between a newbie and a hardcore in the game dota 1," in 2018 Congreso Argentino de Ciencias de la Informtica y Desarrollos de Investigacin (CACIDI), Nov 2018, pp. $1-4$.

[3] M. J. C. Samonte, L. A. Vea, R. M. C. San Jose, A. John V. Lagoy, C. E. P. Manlapid, and A. Martyn P. Perez, "An affect detector model for gamers on a role-playing game through mouse movements," in 2018 International Conference on Information and Communication Technology Convergence (ICTC), Oct 2018, pp. 489-494.

[4] K. J. Shim, K. Hsu, S. Damania, C. DeLong, and J. Srivastava, "An exploratory study of player and team performance in multiplayer firstperson-shooter games," in 2011 IEEE Third International Conference on Privacy, Security, Risk and Trust and 2011 IEEE Third International Conference on Social Computing, Oct 2011, pp. 617-620.

[5] D. Buckley, K. Chen, and J. Knowles, "Rapid skill capture in a firstperson shooter," IEEE Transactions on Computational Intelligence and AI in Games, vol. 9, no. 1, pp. 63-75, March 2017.

[6] A. Drachen, M. Yancey, J. Maguire, D. Chu, I. Y. Wang, T. Mahlmann, M. Schubert, and D. Klabajan, "Skill-based differences in spatiotemporal team behaviour in defence of the ancients 2 (dota 2)," in 2014 IEEE Games Media Entertainment, Oct 2014, pp. 1-8.

[7] M. Suznjevic, M. Matijasevic, and J. Konfic, "Application context based algorithm for player skill evaluation in moba games," in 2015 International Workshop on Network and Systems Support for Games (NetGames), Dec 2015, pp. 1-6.

[8] K. J. Shim, K. Hsu, and J. Srivastava, "An exploratory study of player performance, motivation, and enjoyment in massively multiplayer online role-playing games," in 2011 IEEE Third International Conference on Privacy, Security, Risk and Trust and 2011 IEEE Third International Conference on Social Computing, Oct 2011, pp. 135-140.

[9] _ - "Effects of mentoring on player performance in massively multiplayer online role-playing games (mmorpgs)," in 2011 International Conference on Advances in Social Networks Analysis and Mining, July 2011, pp. 561-562.

[10] E. Cambria, "Affective computing and sentiment analysis," IEEE Intelligent Systems, vol. 31, no. 2, pp. 102-107, Mar 2016.

[11] M. Aung, V. Bonometti, A. Drachen, P. Cowling, A. V. Kokkinakis, C. Yoder, and A. Wade, "Predicting skill learning in a large, longitudinal moba dataset," in 2018 IEEE Conference on Computational Intelligence and Games (CIG), Aug 2018, pp. 1-7.
[12] M. H. Schiller, G. Wallner, C. Schinnerl, A. M. Calvo, J. Pirker, R. Sifa, and A. Drachen, "Inside the group: Investigating social structures in player groups and their influence on activity," IEEE Transactions on Games, pp. 1-1, 2018.

[13] H. Z. Tan, L. A. Slivovsky, and A. Pentland, "A sensing chair using pressure distribution sensors," IEEE/ASME Transactions on Mechatronics, vol. 6 , no. 3, pp. 261-268, Sep. 2001

[14] J. Meyer, B. Arnrich, J. Schumm, and G. Troster, "Design and modeling of a textile pressure sensor for sitting posture classification," IEEE Sensors Journal, vol. 10, no. 8, pp. 1391-1398, Aug 2010.

[15] R. Kumar, A. Bayliff, D. De, A. Evans, S. K. Das, and M. Makos, "Care-chair: Sedentary activities and behavior assessment with smart sensing on chair backrest," in 2016 IEEE International Conference on Smart Computing (SMARTCOMP), May 2016, pp. 1-8.

[16] B. Arnrich, C. Setz, R. La Marca, G. Trster, and U. Ehlert, "What does your chair know about your stress level?" IEEE Transactions on Information Technology in Biomedicine, vol. 14, no. 2, pp. 207-214, March 2010.

[17] M. Park, Y. Song, J. Lee, and J. Paek, "Design and implementation of a smart chair system for iot," in 2016 International Conference on Information and Communication Technology Convergence (ICTC), Oct 2016, pp. 1200-1203.

[18] L. Martins, I. Marques, R. Almeida, J. Costa, C. Quaresma, and P. Vieira, "The influence of wearing a wallet in your sitting posture a quantitative study using an intelligent chair," in 2017 IEEE 5th Portuguese Meeting on Bioengineering (ENBENG), Feb 2017, pp. 1-4.

[19] T. Labeodan, K. Aduda, W. Zeiler, and F. Hoving, "Experimental evaluation of the performance of chair sensors in an office space for occupancy detection and occupancy-driven control," Energy and Buildings, vol. 111, pp. 195-206, 2016.

[20] T. Koivistoinen, S. Junnila, A. Varri, and T. Koobi, "A new method for measuring the ballistocardiogram using emfi sensors in a normal chair," in The 26th Annual International Conference of the IEEE Engineering in Medicine and Biology Society, vol. 1, Sep. 2004, pp. 2026-2029.

[21] S. Junnila, A. Akhbardeh, A. Varri, and T. Koivistoinen, "An emfifilm sensor based ballistocardiographic chair: performance and cycle extraction method," in IEEE Workshop on Signal Processing Systems Design and Implementation, 2005., Nov 2005, pp. 373-377.

[22] B. Ahn, Y. Noh, and D. Jeong, "Smart chair based on multi heart rate detection system," in 2015 IEEE SENSORS, Nov 2015, pp. 1-4.

[23] R. Tibshirani, "Regression shrinkage and selection via the lasso," Journal of the Royal Statistical Society: Series B (Methodological), vol. 58, no. 1, pp. 267-288, 1996.

[24] J. Kuha, "Aic and bic: Comparisons of assumptions and performance," Sociological methods \& research, vol. 33, no. 2, pp. 188-229, 2004.

[25] D. G. Kleinbaum, K. Dietz, M. Gail, M. Klein, and M. Klein, Logistic regression. Springer, 2002.

[26] S.-i. Amari and S. Wu, "Improving support vector machine classifiers by modifying kernel functions," Neural Networks, vol. 12, no. 6, pp. 783-789, 1999

[27] A. Liaw, M. Wiener et al., "Classification and regression by randomforest," $R$ news, vol. 2, no. 3, pp. 18-22, 2002.

[28] T. M. Cover, P. E. Hart et al., "Nearest neighbor pattern classification," IEEE transactions on information theory, vol. 13, no. 1, pp. 21-27, 1967.

[29] H. Zhang, "The optimality of naive bayes," $A A$, vol. 1, no. 2, p. 3, 2004.

[30] C. K. Williams and C. E. Rasmussen, Gaussian processes for machine learning. MIT Press Cambridge, MA, 2006, vol. 2, no. 3.

[31] J. Fan, S. Upadhye, and A. Worster, "Understanding receiver operating characteristic (roc) curves," Canadian Journal of Emergency Medicine, vol. 8, no. 1, pp. 19-20, 2006

[32] A. Smerdov, A. Kishkun, R. Shaniiazov, A. Somov, and E. Burnaev, "Understanding cyber athletes behaviour through a smart chair: CS:GO and monolith team scenario," in 2019 IEEE 5th World Forum on Internet of Things (WF-IoT) (WF-IoT 2019), Limerick, Ireland, Apr. 2019, pp. 973-978.

[33] E. V. Burnaev and I. S. Men'shikov, "A model of the functional state of participants of laboratory markets," Journal of Computer and Systems Sciences International, vol. 48, no. 6, p. 1002, Dec 2009. [Online]. Available: https://doi.org/10.1134/S1064230709060148

[34] V. Ishimtsev, A. Bernstein, E. Burnaev, and I. Nazarov, "Conformal k-nn anomaly detector for univariate data streams," in Proceedings of the Sixth Workshop on Conformal and Probabilistic Prediction and Applications, ser. Proceedings of Machine Learning Research, 
A. Gammerman, V. Vovk, Z. Luo, and H. Papadopoulos, Eds., vol. 60. Stockholm, Sweden: PMLR, 13-16 Jun 2017, pp. 213-227. [Online]. Available: http://proceedings.mlr.press/v60/ishimtsev17a.html

[35] E. Burnaev, I. Koptelov, G. Novikov, and T. Khanipov, "Automatic construction of a recurrent neural network based classifier for vehicle passage detection," in Proc. SPIE, vol. 10341, 2017, pp. 10341 10341 - 6. [Online]. Available: https://doi.org/10.1117/12.2268706

[36] A. Kuleshov, A. Bernstein, and E. Burnaev, "Kernel regression on manifold valued data," in Proceedings of IEEE 5th International Conference on Data Science and Advanced Analytics, 2018, pp. 120-129. 